\title{
Characterization of viral genomic mutations in novel influenza A (H7N9)-infected patients: the association between oseltamivir-resistant variants and viral shedding duration
}

\author{
Renke Chen ${ }^{1}$. Qianda Zou ${ }^{2,3} \cdot$ Guoliang Xie ${ }^{2,3} \cdot \mathrm{Fei} \mathrm{Yu}^{2,3} \cdot$ Xianzhi Yang $^{2,3} \cdot$ Lingyong Cao $^{1} \cdot$ Zhaoxia Huo $^{4}$. \\ Shufa Zheng ${ }^{2,3}$
}

Received: 26 January 2019 / Accepted: 14 June 2019 / Published online: 13 July 2019

๑) Springer Science+Business Media, LLC, part of Springer Nature 2019

\begin{abstract}
Since February 2013, human infections with the novel influenza A H7N9 virus have occurred in eastern China. It is important to detect mutations in viral genes and analyze the clinical features of patients and viral shedding duration related to neuraminidase inhibitor (NAI) resistance. We collected clinical specimens from 31 hospitalized H7N9 patients and sequenced NA, PB2, HA, and M gene fragments. Of the 31 identified patients, 7 (22.6\%) carried the R292K substitution in NA, 30 (96.8\%), 3 (9.7\%), and 5 (16.1\%) carried E627K, Q591K, and D701N mutations in PB2, respectively, and 2 (6.5\%) carried both E627K and D701N mutations in PB2. All 26 identified patients harbored Q226L mutations and possessed only a single arginine (R) at cleavage sites in the HA and a S31N mutation in M2. Among 7 NA-R292K mutated patients, 3 died and 4 were discharged. There was no significant difference in the days that patients started oseltamivir treatment after symptom onset between NA-R292K mutant and NA-R292 wild-type patients (median days, 7 vs $6, P=0.374$ ). NA-R292K mutant patients had a significantly longer duration of viral shedding than NA-R292 wild-type patients after oseltamivir treatment (median days, 10 vs $5, P=0.022$ ). The mutation of R292K in NA conferring the potential ability of oseltamivir resistance resulted in prolonged viral duration and poor outcome and should be taken into consideration in the clinical management of infected patients.
\end{abstract}

Keywords Influenza A (H7N9) · Mutation · Neuraminidase (NA) · Oseltamivir resistance $\cdot$ Viral duration

\section{Introduction}

Human infection with the novel avian-origin influenza A (H7N9) virus has been occurring since February 2013, in eastern China [1]. To date, 1220 people have been infected with the influenza A (H7N9) virus, and 494 people have died [2]. Although outbreaks of highly pathogenic avian influenza virus $\mathrm{H} 5$ infection in wild birds and poultry and $\mathrm{H} 7$ subtype

Electronic supplementary material The online version of this article (https://doi.org/10.1007/s11262-019-01678-8) contains supplementary material, which is available to authorized users.

Zhaoxia Huo

zxhuo@zju.edu.cn

Shufa Zheng

zsfzheng@zju.edu.cn

1 School of Basic Medicine, Zhejiang Chinese Medical University, Hangzhou, People's Republic of China

2 Center of Clinical Laboratory, First Affiliated Hospital, School of Medicine, Zhejiang University, 79 Qingchun Road, Hangzhou 310003, People's Republic of China
Key Laboratory of Clinical In Vitro Diagnostic Techniques of Zhejiang Province, Hangzhou, People's Republic of China

4 Experimental Teaching Center, School of Basic Medical Sciences, Zhejiang University, 866 Yuhangtang Road, Hangzhou 310058, People's Republic of China 
infections in poultry have occurred worldwide, most of them are mild or asymptomatic in humans, except for death associated with $\mathrm{H} 5 \mathrm{~N} 1$ and H7N7 subtype infections [3-5]. Although in the early stage of illness, the genetic analysis of several patients' isolates has indicated that high pathogenicity of avian-origin H7N9 virus in humans, but not poultry, is more likely to result from viral genome variation $[1,6]$; the viral gene variation of long-term shedding is poorly elucidated. In the virus genome, the mutation of Q226L could increase the binding affinity to a human-type receptor $(\alpha-2,6$-linked sialic acid receptors), and cleavage sites might be associated with alteration of receptor specificity from avian origin to a human-type receptor [7-9]. Previous studies concerning H5N1 or H1N1 led to the concept that PB2-627K or PB2-701N mutations are critical for avian influenza virus adaptation to mammals' efficient replication in mammals [10-12]. Other key amino acids such as $591 \mathrm{~K}$ of PB2 can compensate for the lack of lysine at position 627 and confer efficient viral replication to some H1N1 and H5N1 viruses in mammals [13].

Effective antiviral drugs are essential for the early control of an influenza virus pandemic and the spread of infection [14, 15]. There are two classes of antivirals available for influenza prophylaxis and treatment, M2-ion channel blockers adamantanes (amantadine and rimantadine), and neuraminidase (NA) inhibitors (oseltamivir and zanamivir) [16]. The possible threat posed by amantadine and rimantadine-resistant and neuraminidase (NA) inhibitor-resistant influenza viruses has been verified in H1N1 and H5N1 [16-18]. Mutations in S31N in the M2 gene and R292K in the NA gene were noted in several patients' isolates $[1,6]$, suggesting that the emergence of drug-resistant variants should be closely monitored. Therefore, continuous monitoring of the viral gene mutation in specimens originating from one patient with different specimen types and time points is important in understanding the determinants of virulence and transmissibility. In this study, we collected a total of 198 throat swabs, sputum specimens, and stool specimens from 31 patients with laboratory-confirmed H7N9 infection daily after hospital admission whenever available. We noted mutations in the NA, PB2, HA, and M2 genes from 113 clinical specimens whose gene sequences were amplified and sequenced successfully. We summarized the emergence frequency of each substitution in the related genes. Furthermore, we analyzed the viral shedding duration before or after oseltamivir treatment in NA-R292K mutant and NA-R292 wild-type patients.

\section{Materials and methods}

\section{Clinical specimen and data collection}

A total of 31 hospitalized patients with laboratory-confirmed H7N9 virus infection between March 19 and May 31, 2013, in Zhejiang, China, were enrolled. Clinical specimens including throat swabs, sputum samples, and stool samples were collected from patients every day if available and then detected by TaqMan real-time PCR using M, H7, and N9 gene primers as previously described [6]. H7N9-positive specimens were frozen at $-80{ }^{\circ} \mathrm{C}$ and ready for the subsequent experiments. Clinical data were recorded and collected as described in our previous study [6].

\section{RNA extraction, RT-PCR, and sequencing}

Viral RNA extraction from clinical specimens was performed using RNeasy Plus Mini Kit (Qiagen, Germany) following the manufacturer's instructions. Two-step reverse-transcription (RT-PCR) amplifications were performed using the QuantiTect Reverse Transcription Kit (Qiagen, Germany) and the HotStarTaq Plus Master Mix Kit (Qiagen, Germany) to amplify the partial gene segments spanning positions 275 and 292 in NA, positions 526, 591, 627 and 701 in PB2, position 226 in HA, the cleavage site in HA, and position 31 in the M2 gene. Primers are shown in Table S1 in Supplementary material. PCR products were then purified and directly sequenced by the Sanger method to examine the related site mutations.

\section{Gene sequence analysis}

Partial gene segment sequences obtained by direct sequencing were analyzed together with virus sequence data (A/ Shanghai/1/2013; A/Shanghai/2/2013; A/Anhui/1/2013 and A/Zhejiang/1/2013) available from GenBank. Bioedit 7 was used for alignment and analysis of amino acid residues. Our gene sequences were deposited in GISAID databases (accession numbers EPI1443452-EPI1443457, EPI1443872, EPI1443873, EPI1443875, EPI1443938, EPI1443962, EPI1443992, EPI1444004-EPI1444008, EPI1444011, EPI1444018, EPI1444024, EPI1444025, EPI1444054, EPI1444063, EPI1444234-EPI1444240, EPI1444242).

\section{Statistical analysis}

Categorical variables were analyzed using the Chi-square test or the Fisher exact test, as appropriate. Continuous variables were summarized as medians with interquartile ranges and the Mann-Whitney $U$ test was used for analysis. $P<0.05$ was considered statistically significant. SPSS for Windows (version 16.0; SPSS) was used for statistical analysis.

\section{Results}

\section{Critical amino acid residue mutated proportion}

We analyzed the gene sequences amplified directly from clinical specimens together with 4 virus sequences using 
Bioedit 7. All the sequences were highly similar in the amplified regions except for the variability at related positions such as R292K in NA (see Supplementary Fig S1).

We collected a total of 198 specimens, among which 113 specimens, including 25 throat swabs, 75 sputum samples, and 13 stool samples, were amplified and sequenced for NA and PB2 gene fragments successfully. Of 113 specimens from 31 patients, we identified $93(82.3 \%)$ specimens from $30(96.8 \%)$ patients harboring the E627K mutation in the PB2 gene (PB2-E627K), 8 (7.1\%) from 3 (9.7\%) patients harboring PB2-Q591K, 6 (5.3\%) from $5(16.1 \%)$ patients harboring PB2-D701N, and $2(1.8 \%)$ from $2(6.5 \%)$ patients harboring both PB2-E627K and PB2-D701N, which had not been found previously in H7N9 viral isolates. In addition, 7 (22.6\%) of 31 patients harbored the R292K mutation in the NA gene (NA-R292K), and the mutated sequences of the specimens included 4 throat swabs, 9 sputum samples, and 1 stool sample (Table 1).

We detected mutations in HA gene fragments spanning position 226 or the cleavage site as well as the M2 gene fragment spanning position 31 in 63 specimens from 26 patients. We identified that all $(100 \%)$ of the specimens carried the Q226L substitution in the HA gene and S31N in the M2 gene; all (100\%) possessed only a single arginine (R) at the cleavage site in HA (Table 1).

\section{Clinical features and outcomes of the patients}

Given that nearly all patients in our study harbored mutations in the HA, M2, and PB2 genes, we labeled the patients as two groups: NA-R292K mutant patients and NA-R292 wild-type patients. The clinical characteristics of the NAR292K mutant group of patients are shown in Table 2. For 7 NA-R292K mutant patients, with a median age of 70 (range
50 to 79 years), 3 were male (Table 2). Four of them had one or more underlying diseases of hypertension (patient 5 , patient 3 and patient 6), diabetes (patient 5 and patient 6 ), coronary heart disease (patient 5 and patient 3 ), chronic renal disease (patient 5), and lymphoma (patient 2). All 7 patients developed acute respiratory distress syndrome (ARDS), and 6 required mechanical ventilation (Table 2). All patients received oseltamivir treatment, and 2 of them (patient 6 and patient 7) also received peramivir therapy (300 mg every day). The patients were given $75 \mathrm{mg}$ to $150 \mathrm{mg}$ of oral oseltamivir TID after tests for H7N9 virus were positive, starting a median of 7 days (range 4 to 9 days) after symptom onset. Three patients died, and four patients were discharged (Table 2).

A comparison of the clinical symptoms and severity of illness between the NA-R292K mutant and NA-R292 wildtype patients is shown in Table 3. Compared with NA-R292 wild-type patients, patients with the NA-R292K mutation were not significantly older (median age, 70 vs 59 years, $P=0.103$ ). The days of initiation of oseltamivir treatment after symptom onset had no significant difference (median days, 7 vs $6, P=0.374$ ) or very close durations of oseltamivir reception (median days, 11 vs $12.5, P=0.831$ ) between the two groups of patients. The median time of hospital admission after symptom onset for the two groups of patients was also very similar (median days, 7 vs $6, P=0.470$ ), and NA-R292K mutant patients were more likely to have longer hospital days, but this finding was not statistically significant (median days, 31 vs $19, P=0.741$ ).

The percentage of patients who developed ARDS, shock and acute kidney injury during the illness between the two groups were not statistically significant $(P=0.076,0.173$ and 0.063 , respectively). Over $50 \%$ of the patients in the two groups had underlying conditions $(57.1 \%$ vs $62.5 \%$,
Table 1 The proportion of substitutions at critical positions in the proteins of human H7N9 virus in clinical specimens from patients

\begin{tabular}{|c|c|c|c|c|c|}
\hline \multirow[t]{2}{*}{ Protein substitution } & \multirow{2}{*}{$\begin{array}{l}\text { Patient }^{\mathrm{a}} \\
\text { No. }(\%)\end{array}$} & \multicolumn{4}{|l|}{ Specimen $^{\text {b }}$} \\
\hline & & $\begin{array}{l}\text { Throat swab } \\
\text { No. }(\%)\end{array}$ & $\begin{array}{l}\text { Sputum } \\
\text { No. }(\%)\end{array}$ & $\begin{array}{l}\text { Stool } \\
\text { No. }(\%)\end{array}$ & $\begin{array}{l}\text { Total } \\
\text { No. }(\%)\end{array}$ \\
\hline NA-R292K & $7 / 31(22.6)$ & $4 / 25(16.0)$ & $9 / 75(12.0)$ & $1 / 13(7.7)$ & $14 / 113(12.4)$ \\
\hline PB2-K526R & $0 / 31(0.0)$ & $0 / 25(0.0)$ & $0 / 75(0.0)$ & $0 / 13(0.0)$ & $0 / 113(0.0)$ \\
\hline PB2-Q591K & $3 / 31(9.7)$ & $2 / 25(8.0)$ & $6 / 75(8.0)$ & $0 / 13(0.0)$ & $8 / 113(7.1)$ \\
\hline PB2-E627K & 30/31 (96.8) & $19 / 25(76.0)$ & $63 / 75(84.0)$ & $11 / 13(84.6)$ & $93 / 113(82.3)$ \\
\hline PB2-D701N & $5 / 31(16.1)$ & $1 / 25(4.0)$ & $4 / 75(5.3)$ & $1 / 13(7.7)$ & $6 / 113(5.3)$ \\
\hline PB2-E627K and D701N & $2 / 31(6.5)$ & $0 / 25(0.0)$ & $1 / 75(1.3)$ & $1(7.7)$ & $0 / 113(0.0)$ \\
\hline HA-Q226L & $26 / 26(100)$ & $8 / 8(100)$ & $51 / 51(100)$ & $4 / 4(100)$ & $63 / 63(100)$ \\
\hline HA cleavage site & $26 / 26(100)$ & $8 / 8(100)$ & $51 / 51(100)$ & 4/4 (100) & $63 / 63(100)$ \\
\hline $\mathrm{M} 2-\mathrm{S} 31 \mathrm{~N}$ & $26 / 26(100)$ & $8 / 8(100)$ & $51 / 51(100)$ & 4/4 (100) & $63 / 63(100)$ \\
\hline
\end{tabular}


Table 2 Clinical features and virological findings of patients with a substitution at position 292 in NA

\begin{tabular}{|c|c|c|c|c|c|c|c|}
\hline & Patient 1 & Patient 2 & Patient 3 & Patient 4 & Patient 5 & Patient $6^{\mathrm{a}}$ & Patient $7^{\mathrm{a}}$ \\
\hline Age (years) & 70 & 62 & 73 & 76 & 66 & 79 & 50 \\
\hline Sex & Male & Female & Female & Male & Male & Female & Female \\
\hline $\begin{array}{l}\text { Underlying } \\
\text { disease }\end{array}$ & None & Lymphoma & $\begin{array}{l}\text { Hypertension, } \\
\text { CHD }\end{array}$ & None & $\begin{array}{l}\text { Hypertension, } \\
\text { Diabetes, } \\
\text { CHD, CRD }\end{array}$ & $\begin{array}{l}\text { Hypertension, } \\
\text { Diabetes, } \\
\text { CHD }\end{array}$ & None \\
\hline ARDS & Yes & Yes & Yes & Yes & Yes & Yes & Yes \\
\hline $\begin{array}{l}\text { Hospitalized } \\
\text { (days) }\end{array}$ & 2 & 6 & 37 & 31 & 62 & 30 & 41 \\
\hline Outcome & Died & Died & Died & Discharge & Discharge & Discharge & Discharge \\
\hline $\begin{array}{l}\text { Time between } \\
\text { onset of symp- } \\
\text { toms and need } \\
\text { for mechanical } \\
\text { ventilation } \\
\text { (days) }\end{array}$ & 6 & 3 & 7 & N/A & 3 & 7 & 8 \\
\hline $\begin{array}{l}\text { Time between } \\
\text { mechanical } \\
\text { ventilation and } \\
\text { death (days) }\end{array}$ & 3 & 8 & 34 & N/A & N/A & N/A & N/A \\
\hline $\begin{array}{l}\text { Time between } \\
\text { onset of } \\
\text { symptoms and } \\
\text { initiation of } \\
\text { oseltamivir } \\
\text { (days) }\end{array}$ & 7 & 6 & 6 & 9 & 4 & 8 & 8 \\
\hline $\begin{array}{l}\text { Oseltamivir } \\
\text { given (days } \\
\text { after symp- } \\
\text { toms, dosage } \\
\text { daily) }\end{array}$ & Day 6, $300 \mathrm{mg}$ & Day 6, $300 \mathrm{mg}$ & Day $5,150 \mathrm{mg}$ & Day $8,150 \mathrm{mg}$ & Day 3,150 mg & Day $7,150 \mathrm{mg}$ & Day $7,150 \mathrm{mg}$ \\
\hline $\begin{array}{l}\text { Time of } \\
\text { specimens } \\
\text { collection after } \\
\text { oseltamivir } \\
\text { given (days) }\end{array}$ & Day $1-2$ & Day 6-11 & Day $1-3,5$ & Day 0-4 & Day 3-10 & Day $2-10$ & Day $1-4,7-12$ \\
\hline $\begin{array}{l}\text { Emergence of } \\
\text { the subsitu- } \\
\text { tion of R292K } \\
\text { in NA after } \\
\text { oseltamivir } \\
\text { given }\end{array}$ & $\begin{array}{l}\text { Day } 2 \text { in throat } \\
\text { swab; Day } 2 \text { in } \\
\text { sputum }\end{array}$ & $\begin{array}{l}\text { Day } 8 \text { in throat } \\
\text { swab; Day } \\
7-10 \text { in spu- } \\
\text { tum }\end{array}$ & Day 2 in stool & $\begin{array}{l}\text { Day } 1 \text { in throat } \\
\text { swab; }\end{array}$ & $\begin{array}{l}\text { Day } 10 \text { in } \\
\text { sputum }\end{array}$ & $\begin{array}{l}\text { Day } 5 \text { in } \\
\text { throat swab; } \\
\text { Day } 7,9 \text { in } \\
\text { sputum }\end{array}$ & Day 10 in sputum \\
\hline
\end{tabular}

$A R D S$ acute respiratory distress syndrome, $C H D$ coronary heart disease, $C R D$ chronic renal disease

${ }^{\text {a }}$ Patient also received peramivir therapy (300 mg every day)

$P=0.789)$, and received mechanical ventilation $(85.7 \%$ vs $50.0 \%, P=0.092)$. A total of $57.1 \%$ of patients out of the NA-R292K mutant group compared with $25 \%$ out of the NA-R292 wild-type group received extracorporeal membrane oxygenation (ECMO). In total, $42.9 \%$ of patients out of the NA-R292K mutant group, compared with $20.8 \%$ out of the NA-R292 wild-type group, received glucocorticoid therapy. However, there was no significant difference between the two groups according to statistical analysis. NA-R292K mutant patients were more likely to have higher acute physiology and chronic health evaluation II
(APACHE-II) scores compared with NA-R292 wild-type patients $(P=0.039)$. Although not statistically significance, it seems that the NA-R292K mutant group had higher lethality $(42.9 \%$ vs $12.5 \%, P=0.074)$, which is shown in Table 3 and Fig S2 in Supplementary material.

\section{Virological findings of the patients}

We noted that oseltamivir resistance was associated with the R292K mutation (position 292 with N2 numbering; position 294 with N9 numbering) in the NA protein in 14 specimens 
Table 3 Comparison of clinical symptoms and severity of illness between NA-R292K mutant and NA-R292 wild-type patients

\begin{tabular}{lccc}
\hline & $\begin{array}{l}\text { NA-R292K } \\
\text { mutant }(n=7) \\
\text { No. }(\%)\end{array}$ & $\begin{array}{l}\text { NA-R292 wild } \\
\text { type }(n=24) \\
\text { No. }(\%)\end{array}$ & $P$ value \\
\hline Male sex & $3(42.9)$ & $15(62.5)$ & 0.354 \\
Age (year) & $70(66-79)$ & $59(34-86)$ & 0.103 \\
Underlying conditions & $4(57.1)$ & $15(62.5)$ & 0.798 \\
Extracorporeal membrane oxygenation & $4(57.1)$ & $6(25.0)$ & 0.109 \\
Mechanical ventilation & $6(85.7)$ & $12(50.0)$ & 0.092 \\
ARDS & $7(100.0)$ & $16(66.7)$ & 0.076 \\
Shock & $4(57.1)$ & $7(29.2)$ & 0.173 \\
Acute kidney injury & $4(57.1)$ & $5(20.8)$ & 0.063 \\
Glucocorticoid therapy & $3(42.9)$ & $5(20.8)$ & 0.241 \\
Antivirals (> 1) & $2(28.6)$ & $3(12.5)$ & 0.309 \\
Time between onset of symptoms and initiation of & $7(4-9)$ & $6(2-16)$ & 0.374 \\
$\quad$ oseltamivir (days) & & & \\
Duration of oseltamivir given (days) & $11(4-23)$ & $12.5(1-20)$ & 0.831 \\
Time between symptom onset and admission (days) & $7(4-10)$ & $6(1-14)$ & 0.470 \\
Hospitalized (days) & $31(2-62)$ & $19(5-52)$ & 0.741 \\
APACHE-II score & $32(16-41)$ & $19(10-40)$ & 0.039 \\
Death & $3(42.9)$ & $3(12.5)$ & 0.074 \\
\hline
\end{tabular}

ARDS acute respiratory distress syndrome, APACHE-II Acute Physiology and Chronic Health Evaluation II from 7 patients (Table 1). Interestingly, the emergence of the R292K mutation was detected on different days after oseltamivir treatment. The R292K mutations emerged from 7 days after oseltamivir treatment in 3 patients (patient 2 , patient 5 and patient 7). The emergence of the R292K mutation was identified on day 1-2 after oseltamivir treatment was initiated from patient 1 , patient 3 , and patient 4 (Table 2). Mutations in Q591K, E627K, and D701N of the PB2 gene were also detected in those specimens (see Table S2 in Supplementary material).

Since the function of the R292K mutation is associated with oseltamivir resistance, we analyzed the virus shedding duration between NA-R292K mutant patients and NA-R292 wild-type patients to compare and confirm whether the effects of oseltamivir therapy were different. We presumed that virus positivity was initiated at the beginning of symptom onset as in a previous study (Ling et al. [14]), and we summarized the days of virus positivity before and after oseltamivir treatment until the virus was negative (Fig. 1). The statistical analysis showed that the time from symptom onset to initiation of oseltamivir between NA-R292K mutant patients (patient 1 and patient 2 with continuous viral positive shedding until death were out of our analysis) and NA-R292 wild-type patients had no significant difference (median days, 8 vs $6, P=0.395$ ), but NA-R292K mutants were more likely to prolong the viral shedding duration after oseltamivir treatment than NA-R292 wild-type patients (median days, 10 vs 5, $P=0.022$ ). In addition, NA-R292 wild-type patients had a significantly shorter time from symptom onset to virus transfer (median days, 17 vs 10.5,
$P=0.016)$ and a shorter duration between symptom onset and outcome (median days, 43 vs 24.5, $P=0.040$ ) (Fig. 2).

\section{Discussion}

In this study, we noted the mutations in the gene fragments of novel avian-origin influenza A (H7N9) virus, and identified that the Q226L mutation in the HA gene, S31N in the M2 gene, and the deletion of repeated arginine (R) in the HA cleavage site were the most frequently emerged mutations in H7N9 virus-infected patients (100\%). The emergence of E627K (30 of 31 patients, 96.8\%) substitution seemed to be more frequent than Q591K (3 of 31 patients, 9.7\%) and D701N (5 of 31 patients, 16.1\%) in PB2, and mutations emerging simultaneously at both the 627 and 701 sites in PB2 rarely occurred (only 2 among 113 specimens). Seven (22.6\%) of 31 patients harbored R292K mutations in the NA gene. Prolonged viral shedding duration was reported in R292K mutant patients, even after receipt of oseltamivir therapy. Although there were no statistically significant differences in clinical features between those with or without the NA-R292K mutation because of limited numbers, the severe illness conditions and high lethality of NA-R292K mutant patients should also be considered.

In humans, the clinical outcome of influenza varies from mild to severe or fatal. Viral factors can be important in determining disease severity [19]. Unlike other types of avian influenza virus affecting human beings, infection with the novel influenza A (H7N9) virus in humans did 


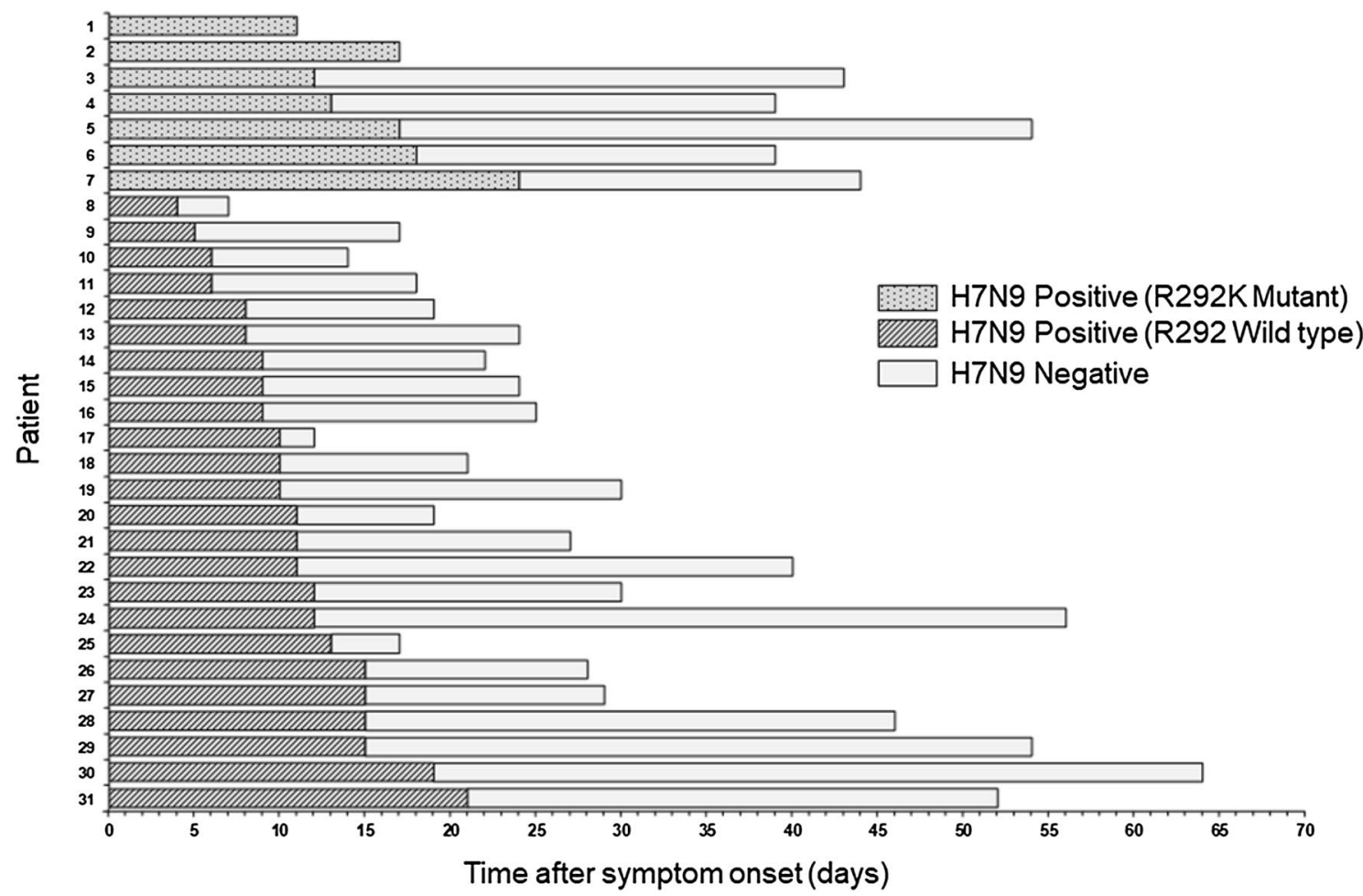

Fig. 1 The duration of viral positivity in the course of the disease of H7N9-infected patients. End point of every bar: patient outcome (died, or discharge, or still under treatment by the time of May 31, 2013)

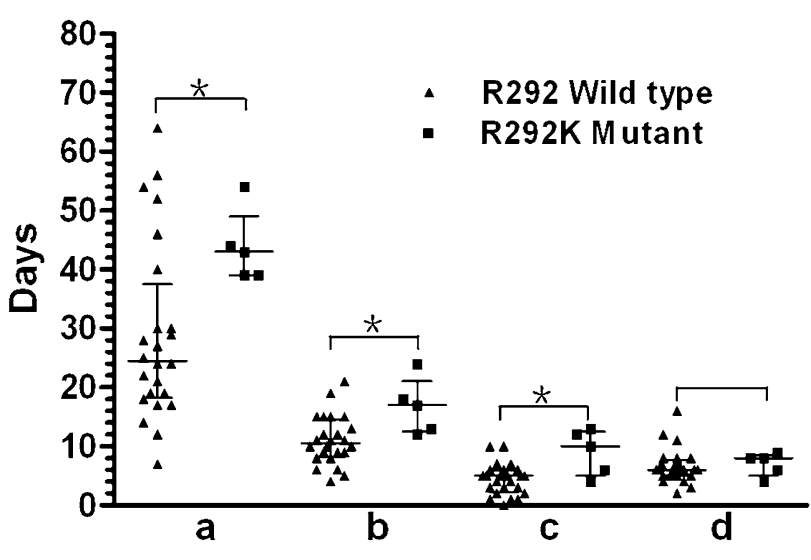

Fig. 2 Comparison of viral positive days sustained in clinical specimens of NA-R292K mutant patients and NA-R292 wild-type patients. Two NA-R292K mutant patients (patient 1 and patient 2) were excluded for the analysis because it was difficult to estimate the viral positive duration because we did not detect the viral negative specimens until they died. a Time between symptom onset and outcome (median days, 43 vs $24.5, P=0.016$ ). b Time between symptom onset and initiation of virus negative in specimens (median days, 17 vs $10.5, P=0.040)$. c Time between start of oseltamivir treatment and virus negative in specimens (median days, 10 vs $5, P=0.022$ ). d Time between symptom onset and initiation of oseltamivir treatment (median days, 8 vs $6, P=0.395$ ). ${ }^{*} P<0.05$ not cause an increase in poultry deaths. Therefore, the key concerns about the current outbreak of influenza A (H7N9) virus are how the virus crosses the species barrier. The sequence analysis of H7N9 for several patients by our previous study and Gao's group reported that the Q226L mutation in HA and the lack of a glycosylation site (cleavage site) were more likely to change the avian origin receptor to an alpha 2,6 human-like receptor and might decrease the ability of the virus to be transmitted by air $[1,6]$. The Q226L mutation in HA was also detected in all 63 specimens from 26 patients in our study. The lack of a cleavage site in HA was also found in the H7N9 virus in all specimens. The $100 \%$ emergence frequency of Q226L and cleavage site in HA in our current study indicated that the emergence of those viral variants was not a chance happening under certain circumstances. The absence of the multibasic amino acid motif of the HA cleavage site which is a key virulence marker in birds, endows the highly pathogenic avian $\mathrm{H} 5 \mathrm{~N} 1$ and $\mathrm{H} 7 \mathrm{~N} 7$ viruses with multiorgan tropism [19], but whether it is a virulence marker in people needs to be further confirmed [6]. The substitution of Q591K, E627K, or D701N of PB2 is associated with mammalian adaptation and efficient replication and transmission and is found in all $\mathrm{H} 1 \mathrm{~N} 1, \mathrm{H} 2 \mathrm{~N} 2$, and $\mathrm{H} 3 \mathrm{~N} 2$ influenza viruses that infect humans [13, 20, 21]. Mutations in E627K or D701N were also identified 
in cases of infection in the Shanghai, Anhui, and Zhejiang H7N9 isolates $[1,6]$. We found that the mutation of Q591K was also present in the H7N9 virus. In addition, the simultaneous emergence of E627K and D701N identified in our study rarely occurred. The characteristics of the adaptation and efficient replication ability of this virus may enhance H7N9 influenza viruses, contributing to the prolonged duration of viral shedding and severe disease in almost all patients.

The $\mathrm{S} 31 \mathrm{~N}$ mutation in the M2 gene was also noted in all of the patients enrolled, indicating that neuraminidase inhibitors, including oseltamivir, zanamivir, and peramivir have been the main drugs used for antiviral treatment of patients infected with H7N9. We also detected seven patients harboring the neuraminidase inhibitor-resistant associating R292K mutation. The emergence of the R292K mutation was detected from 1 day (range 1-12 days) after oseltamivir treatment. As we could not collect the specimens and detect R292K before oseltamivir treatment, whether the R292K mutation occurred naturally or drug-induced could not be determined. The latest study showed that the emergence of an NA-R292K mutation infected with H7N9 was temporally associated with a high rebound virus load, treatment failure, and a poor clinical outcome [22]. We did not quantify the viral load in this study but did find that the emergence of the NA-R292K mutation delayed the viral shedding duration for the NA-R292K mutant patients, affecting the effects of antiviral therapy. Furthermore, recent and previous studies indicated that early oseltamivir treatment started 2 days before for $\mathrm{H} 7 \mathrm{~N} 9$ or 4 days before for $\mathrm{H} 1 \mathrm{~N} 1$ after disease onset would decrease the virus load or shorten the duration of viral shedding $[14,22]$. In our study, the median initial time of oseltamivir treatment after symptom onset was 7 and 6 days for the two groups of patients. The extended time of hospital visit time for the patients may be one reason for prolonged viral shedding duration.

Disease severity depends on both host and viral factors. Host factors associated with severe complications from influenza include age and underlying comorbidities [19]. Efficient therapies are also critical for advantageous clinical outcomes. We compared the demographic features and clinical characteristics of illness symptoms and antiviral therapies between NA-R292K mutant patients and NA-R292 wild-type patients, all of which had no statistically significant differences except for APACHE-II score, mainly because of the limited numbers of mutated patients.

In conclusion, even if the poor outcomes of seven NAR292K mutant patients resulting from the antiviral-drugresistant mutations are not certain, the emergence of H7N9 variants should be closely monitored, and the R292K mutation conferring the potential ability of oseltamivir resistance should be taken into consideration in the clinical management of infected patients.
Acknowledgements We thank Prof. Kwok-Yung Yuen and Prof. HongLin Chen in the State Key Laboratory of Emerging Infectious Diseases, Department of Microbiology, University of Hong Kong for their advice in study design, and Dr. Weifeng Liang (State Key Laboratory for Diagnosis and Treatment of Infectious Diseases, First Affiliated Hospital, College of Medicine, Zhejiang University, Hangzhou, China) for help with the clinical data collection.

Funding This work was supported by the China National MegaProjects for Infectious Diseases (Grant Number 2017ZX10103008), the National Natural Science Foundation of China (Grant Numbers 81672014,81600300 and 81702079), the Natural Science Foundation of Zhejiang Province (Grant Number LQ 15H100001), the Fundamental Research Funds for the Central Universities (Grant Number 2017QNA7016), and the General research projects of zhejiang education department (Y201738209).

\section{Compliance with ethical standards}

Conflict of interest We declare that all authors have no conflict of interest.

Ethical approval This study conformed to the ethical guidelines of the 1975 Declaration of Helsinki and was approved by the Institutional Review Board of the First Affiliated Hospital of Zhejiang University.

\section{References}

1. Gao R, Cao B, Hu Y, Feng Z, Wang D, Hu W et al (2013) Human infection with a novel avian-origin influenza A (H7N9) virus. N Engl J Med 368:1888-1897

2. Wang X, Jiang H, Wu P, Uyeki T, Feng L, Lai S et al (2017) Epidemiology of avian influenza A H7N9 virus in human beings across five epidemics in mainland China, 2013-17: an epidemiological study of laboratory-confirmed case series. Lancet Infect Dis 17(8):822-832

3. Arzey GG, Kirkland PD, Arzey KE, Frost M, Maywood P, Conaty $S$ et al (2012) Influenza virus A (H10N7) in chickens and poultry abattoir workers, Australia. Emerg Infect Dis 18(5):814-816

4. Lai S, Qin Y, Cowling BJ, Ren X, Wardrop NA, Gilbert M (2016) Global epidemiology of avian influenza A H5N1 virus infection in humans, 1997-2015: a systematic review of individual case data. Lancet Infect Dis 6(7):e108-e118

5. Fouchier RA, Schneeberger PM, Rozendaal FW, Broekman JM, Kemink SA, Munster V et al (2004) Avian influenza A virus (H7N7) associated with human conjunctivitis and a fatal case of acute respiratory distress syndrome. Proc Natl Acad Sci USA 101(5):1356-1361

6. Chen Y, Liang W, Yang S, Wu N, Gao H, Sheng J et al (2013) Human infections with the emerging avian influenza A H7N9 virus from wet market poultry: clinical analysis and characterisation of viral genome. Lancet 381(9881):1916-1925

7. Chen LM, Blixt O, Stevens J, Lipatov AS, Davis CT, Collins BE et al (2012) In vitro evolution of H5N1 avian influenza virus toward human-type receptor specificity. Virology 422(1):105-113

8. Herfst S, Schrauwen EJ, Linster M, Chutinimitkul S, de Wit E Munster VJ et al (2012) Airborne transmission of influenza A/ H5N1 virus between ferrets. Science 336(6088):1534-1541

9. Imai M, Watanabe T, Hatta M, Das SC, Ozawa M, Shinya K et al (2012) Experimental adaptation of an influenza H5 HA confers respiratory droplet transmission to a reassortant $\mathrm{H} 5 \mathrm{HA} / \mathrm{H} 1 \mathrm{~N} 1$ virus in ferrets. Nature 486(7403):420-428 
10. Hatta M, Gao P, Halfmann P, Kawaoka Y (2001) Molecular basis for high virulence of Hong Kong H5N1 influenza A viruses. Science 293(5536): 1840-1842

11. Li Z, Chen H, Jiao P, Deng G, Tian G, Li Y et al (2005) Molecular basis of replication of duck H5N1 influenza viruses in a mammalian mouse model. J Virol 79(18):12058-12064

12. Subbarao EK, London W, Murphy BR (1993) A single amino acid in the PB2 gene of influenza A virus is a determinant of host range. J Virol 67(4):1761-1764

13. Yamada S, Hatta M, Staker BL, Watanabe S, Imai M, Shinya $\mathrm{K}$ et al (2010) Biological and structural characterization of a host-adapting amino acid in influenza virus. PLoS Pathog 6(8):e1001034

14. Ling LM, Chow AL, Lye DC, Tan AS, Krishnan P, Cui L et al (2010) Effects of early oseltamivir therapy on viral shedding in 2009 pandemic influenza A (H1N1) virus infection. Clin Infect Dis 50(7):963-969

15. Sugaya N, Tamura D, Yamazaki M, Ichikawa M, Kawakami C, Kawaoka A et al (2008) Comparison of the clinical effectiveness of oseltamivir and zanamivir against influenza virus infection in children. Clin Infect Dis 47(3):339-345

16. Yen HL, Ilyushina NA, Salomon R, Hoffmann E, Webster RG, Govorkova EA (2007) Neuraminidase inhibitor-resistant recombinant A/Vietnam/1203/04 (H5N1) influenza viruses retain their replication efficiency and pathogenicity in vitro and in vivo. $\mathrm{J}$ Virol 81(22):12418-12426

17. Hayden FG (2006) Antiviral resistance in influenza virusesimplications for management and pandemic response. $\mathrm{N}$ Engl $\mathrm{J}$ Med 354(8):785-788
18. Hayden FG, Belshe RB, Clover RD, Hay AJ, Oakes MG, Soo W (1989) Emergence and apparent transmission of rimantadine-resistant influenza A virus in families. N Engl J Med 321(25):1696-1702

19. Chen H, Wen X, To KK, Wang P, Tse H, Chan JF et al (2010) Quasispecies of the D225G substitution in the hemagglutinin of pandemic influenza $A(H 1 N 1) 2009$ virus from patients with severe disease in Hong Kong China. J Infect Dis 201(10):1517-1521

20. Steel J, Lowen AC, Mubareka S, Palese P (2009) Transmission of influenza virus in a mammalian host is increased by PB2 amino acids $627 \mathrm{~K}$ or 627E/701N. PLoS Pathog 5(1):e1000252

21. Garten RJ, Davis CT, Russell CA, Shu B, Lindstrom S, Balish A et al (2009) Antigenic and genetic characteristics of swine-origin $2009 \mathrm{~A}(\mathrm{H} 1 \mathrm{~N} 1)$ influenza viruses circulating in humans. Science 325(5937):197-201

22. Hu Y, Lu S, Song Z, Wang W, Hao P, Li J et al (2013) Association between adverse clinical outcome in human disease caused by novel influenza A H7N9 virus and sustained viral shedding and emergence of antiviral resistance. Lancet 381(9885):2273-2279

Publisher's Note Springer Nature remains neutral with regard to jurisdictional claims in published maps and institutional affiliations. 